Yeo Jin Kim, MD

Hee Jin Kim, MD

Jae-Hyun Park, MD

Seonwoo Kim, PhD

Sook-Young Woo, MS

Ki-Chang Kwak, BS

Jong Min Lee, $\mathrm{PhD}$

Na-Yeon Jung, MD

Jae Seung Kim, MD

Yearn Seong Choe, $\mathrm{PhD}$

Kyung-Han Lee, MD

Seung Hwan Moon, MD

Jae-Hong Lee, MD

Yun Joong Kim, MD

David J. Werring, $\mathrm{PhD}$, FRCP

Duk L. Na, MD

Sang Won Seo, MD

Correspondence to

Dr. Seo:

sangwonseo@empal.com

Supplemental data at Neurology.org

\title{
Synergistic effects of longitudinal amyloid and vascular changes on lobar microbleeds
}

\section{ABSTRACT}

Objective: To determine whether amyloid and hypertensive cerebral small vessel disease (hCSVD) changes synergistically affect the progression of lobar microbleeds in patients with subcortical vascular mild cognitive impairment (svMCl).

Methods: Among 72 patients with svMCl who underwent brain MRI and $\left[{ }^{11} \mathrm{C}\right]$ Pittsburgh compound $\mathrm{B}(\mathrm{PiB})-\mathrm{PET}, 52$ (72.2\%) completed the third year of follow-up. These patients were evaluated by annual neuropsychological testing, brain MRI, and follow-up PiB-PET.

Results: Over 3 years, 31 of 52 patients (59.6\%) had incident cerebral microbleeds (CMBs) in the lobar and deep regions. Both baseline and longitudinal changes in lacune numbers were associated with increased numbers of lobar and deep microbleeds, while baseline and longitudinal changes in PiB uptake ratio were associated only with the progression of lobar microbleeds, especially in the temporal, parietal, and occipital areas. Regional white matter hyperintensity severity was also associated with regional lobar CMBs in the parietal and occipital regions. There were interactive effects between baseline and longitudinal lacune number and PiB retention on lobar microbleed progression. Increased lobar, but not deep, CMBs were associated with decreased scores in the digit span backward task and Rey-Osterrieth Complex Figure Test.

Conclusions: Our findings suggest that amyloid-related pathology and hCSVD have synergistic effects on the progression of lobar microbleeds, providing new clinical insight into the interaction between amyloid burden and hCSVD on CMB progression and cognitive decline with implications for developing effective prevention strategies. Neurology ${ }^{\circledR}$ 2016;87:1575-1582

\section{GLOSSARY}

$\mathbf{A D}=$ Alzheimer disease; $\mathbf{A D L}=$ activities of daily living; $\mathbf{C A A}=$ cerebral amyloid angiopathy; $\mathbf{C M B}=$ cerebral microbleed; FLAIR = fluid-attenuated inversion recovery; GRE = gradient echo; hCSVD = hypertensive cerebral small vessel disease; PiB = Pittsburgh compound B; RCFT = Rey-Osterrieth Complex Figure Test; SNSB = Seoul Neuropsychological Screening Battery; SUVR = standardized uptake value ratio; $\mathbf{s v M C I}=$ subcortical vascular mild cognitive impairment; $\mathbf{W M H}=$ white matter hyperintensity.

Alzheimer disease (AD) and hypertensive cerebral small vessel disease (hCSVD) are major causes of cognitive impairment in the elderly. ${ }^{1,2}$ There is increasing evidence that $\mathrm{AD}$ pathologies and hCSVD coexist and interact in individuals with cognitive impairment. ${ }^{3}$ Recent amyloid PET studies suggested that approximately $30 \%$ of patients with extensive hCSVD also had amyloid lesions of the brain, showing a relationship between amyloid and hCSVD and their clinical relevance. $^{4,5}$

Cerebral microbleeds (CMBs) have generated a great deal of interest, since they are thought to result from 2 key age-related small vessel pathologies, cerebral amyloid angiopathy (CAA) and hCSVD, which may have different underlying causes and mechanisms. ${ }^{6,7}$ The topography of

\footnotetext{
From the Department of Neurology (Y.J.K.), Chuncheon Sacred Heart Hospital, Hallym University College of Medicine, Chuncheon; Departments of Neurology (H.J.K., D.L.N., S.W.S.) and Nuclear Medicine (Y.S.C., K.-H.L., S.H.M.), Sungkyunkwan University School of Medicine, and Neuroscience Center (H.J.K., D.L.N., S.W.S.), Samsung Medical Center, Seoul; Department of Neurology (J.-H.P.), Semyeong Christianity Hospital, Pohang; Biostatistics Team (S.K., S.-Y.W.), Samsung Biomedical Research Institute; Department of Biomedical Engineering (K.-C.K., J.M.L.), Hanyang University, Seoul; Department of Neurology (N.-Y.J.), Pusan National University Hospital, Pusan National University School of Medicine and Medical Research Institute, Busan; Departments of Nuclear Medicine (J.S.K.) and Neurology (J.-H.L.), University of Ulsan College of Medicine, Asan Medical Center, Seoul; Department of Neurology (Y.J.K.), Ilsong Institute of Life Science, Hallym University, Anyang, Korea; Stroke Research Centre (D.J.W.), Department of Brain Repair and Rehabilitation, UCL Institute of Neurology, Queen Square, London, UK; and Departments of Health Sciences and Technology (D.L.N.) and Clinical Research Design \& Evaluation (S.W.S.), SAIHST, Sungkyunkwan University, Seoul, Korea.

Go to Neurology.org for full disclosures. Funding information and disclosures deemed relevant by the authors, if any, are provided at the end of the article.
} 
Table 1 Baseline and follow-up demographics and imaging characteristics

\begin{tabular}{|c|c|c|}
\hline & Baseline, $n=72$ & Follow-up, $n=52$ \\
\hline Baseline age, $y^{a}$ & $74.0 \pm 6.9$ & $75.9 \pm 7.1$ \\
\hline$\%$ Female $^{\mathrm{b}}$ & 45/72 (62.5) & $35 / 52(67.3)$ \\
\hline \multicolumn{3}{|l|}{ Vascular risk factors ${ }^{b}$} \\
\hline Hypertension & $54 / 72(75)$ & 40/52 (76.9) \\
\hline Diabetes mellitus & $18 / 72(25)$ & $13 / 52(25)$ \\
\hline Hyperlipidemia & 25/72 (34.7) & 17/52 (32.7) \\
\hline \multicolumn{3}{|l|}{$A P O E$ genotype $^{b}$} \\
\hline$\varepsilon 2$ Allele-positive & 11/72 (15.3) & 9/52 (17.3) \\
\hline$\varepsilon 4$ Allele-positive & 19/72 (26.4) & 12/52 (23.1) \\
\hline$M_{M S E}{ }^{a}$ & $26.2 \pm 2.9$ & $24.3 \pm 5.0$ \\
\hline CDR-SOBa & $1.3 \pm 0.8$ & $2.3 \pm 2.2$ \\
\hline Lacunes $^{c}$ & $4(0-28)$ & $5(0-31)$ \\
\hline Global PiB uptake ratio ${ }^{a}$ & $1.47 \pm 0.38$ & $1.52 \pm 0.45$ \\
\hline PiB positivity $(>1.5)^{b}$ & 20/72 (27.8) & 18/54 (з3.3) \\
\hline $\mathrm{CMBs}$, total ${ }^{\mathrm{C}}$ & $1.5(0-77)$ & $2(0-93)$ \\
\hline Lobar CMBs & $1.0(0-39)$ & $1(0-47)$ \\
\hline Frontal & $0(0-6)$ & $0(0-12)$ \\
\hline Parietal & $0(0-13)$ & $0(0-7)$ \\
\hline Temporal & $0(0-14)$ & $0(0-17)$ \\
\hline Occipital & $0(0-10)$ & $0(0-11)$ \\
\hline Deep CMBs & $0(0-48)$ & $0(0-55)$ \\
\hline \multicolumn{3}{|l|}{ WMH severity ${ }^{d}$} \\
\hline Global WMH (0-84) & $31.5 \pm 7.3$ & $34.4 \pm 7.6$ \\
\hline Frontal WMHe (0-38) & $17.3 \pm 4.7$ & $18.8 \pm 4.4$ \\
\hline Parietal WMHf (0-6) & $3.8 \pm 1.3$ & $3.7 \pm 1.3$ \\
\hline Temporal WMH ${ }^{g}(0-6)$ & $2.9 \pm 1.5$ & $3.1 \pm 1.5$ \\
\hline Occipital WMH $(0-8)$ & $3.4 \pm 1.9$ & $3.5 \pm 1.7$ \\
\hline
\end{tabular}

Abbreviations: $\mathrm{CDR}-\mathrm{SOB}=$ Clinical Dementia Rating Sum of Boxes; $\mathrm{CMB}=$ cerebral microbleed; MMSE = Mini-Mental State Examination; PiB = Pittsburgh compound B; $\mathrm{WMH}=$ white matter hyperintensity.

${ }^{\text {a }}$ Continuous variables expressed as mean \pm SD.

${ }^{\mathrm{b}}$ Categorical variables expressed as prevalence (\%).

${ }^{c}$ Continuous variables expressed as median (minimum-maximum) (variables are not normally distributed).

${ }^{\mathrm{d}}$ WMH was measured using the Scheltens $\mathrm{WMH}$ scale.

${ }^{\text {e } F r o n t a l ~ W M H ~ c o n s i s t e d ~ o f ~ f r o n t a l ~ c a p ~ W M H ~(0-2), ~ l o b a r ~ f r o n t a l ~ W M H ~(0-6), ~ a n d ~ b a s a l ~}$ ganglia hyperintensity (0-30).

${ }^{f}$ Parietal WMH was defined as lobar parietal WMH located in the deep and subcortical white matter.

${ }^{9}$ Temporal WMH was defined as lobar temporal WMH located in the deep and subcortical white matter.

h Occipital WMH consisted of occipital cap WMH (0-2) and lobar occipital WMH (0-6).

$\mathrm{CMBs}$ is thought to reflect their underlying etiology ${ }^{8}$; however, recent studies on this issue showed inconsistent results. ${ }^{9}$ Our group previously showed that amyloid burden and hCSVD are synergistically associated with lobar CMBs. ${ }^{10}$ A major limitation of studies to date is that the association between neuroimaging markers and CMBs has only been investigated in cross-sectional studies, ${ }^{10}$ which limits the exploration of cause-effect relationships.

In the current study, we investigated the longitudinal effects of changes in amyloid burden and hCSVD on the progression of lobar and deep CMBs in patients with subcortical vascular mild cognitive impairment (svMCI). We hypothesized that amyloid and hCSVD changes synergistically affect lobar $\mathrm{CMBs}$ changes, while deep $\mathrm{CMB}$ changes are only associated with hCSVD changes. We also hypothesized that changes in CMBs affect cognitive decline.

METHODS Participants. We prospectively recruited 72 patients with svMCI from September 2008 to September 2011 at Samsung Medical Center. Patients with svMCI were diagnosed using the previously described Petersen's Criteria ${ }^{11}$ with the following modifications $s^{5}$ : (1) a subjective cognitive complaint by the patient or his or her caregiver; (2) normal activities of daily living (ADL) score determined clinically and by the instrumental ADL scale; (3) objective cognitive decline below the 16th percentile on the Seoul Neuropsychological Screening Battery (SNSB); (4) no dementia; (5) presence of a subcortical vascular feature defined as a focal neurologic symptom/sign such as corticobulbar sign, pyramidal sign, or parkinsonism; and (6) significant white matter hyperintensity (WMH) on MRI. Significant WMH was defined as a hyperintensity in the periventricular white matter longer than $10 \mathrm{~mm}$ or hyperintensity in the deep white matter lesion more than $25 \mathrm{~mm}$ in maximum diameter on fluid-attenuated inversion recovery (FLAIR) imaging. The clinical characteristics of the patients are shown in table 1 .

Patients were evaluated by neuropsychological testing, laboratory testing, and MRI as described in a previous study. ${ }^{5}$ Brain MRI confirmed the absence of structural lesions, including territorial cerebral infarction, brain tumor, hippocampal sclerosis, and vascular malformation.

Standard protocol approvals, registrations, and patient consents. Written informed consent was obtained from each patient. The Institutional Review Board of the Samsung Medical Center approved the study protocol.

Neuropsychological tests. All patients underwent neuropsychological testing using the SNSB. ${ }^{12}$ This battery includes quantitative tests, including digit span (forward and backward), the Korean version of the Boston Naming Test, the Rey-Osterrieth Complex Figure Test (RCFT; copying, immediate, and 20-minute delayed recall, and recognition), the Seoul Verbal Learning Test (3 learning-free recall trials of 12 words, a 20-minute delayed recall trial for these 12 items, and a recognition test), the phonemic and semantic Controlled Oral Word Association Test, Stroop Test (word and color reading of 112 items during a 2-minute period), the Mini-Mental State Examination, and the Clinical Dementia Rating Sum of Boxes.

$\left[{ }^{11} \mathrm{C}\right]$ Pittsburgh compound B (PiB)-PET imaging. All patients underwent $\left[{ }^{11} \mathrm{C}\right]$ PiB-PET scanning at Samsung Medical Center or Asan Medical Center with identical settings using a Discovery STe PET/CT scanner (GE Medical Systems, Milwaukee, WI). The detailed radiochemistry profiles, scanning 
protocols, and $\mathrm{PiB}$ data analysis are described in appendix e-1 at Neurology.org and were based on a previous study. ${ }^{5}$ To measure $\mathrm{PiB}$ retention, we used the cerebral cortical region-to-cerebellum uptake ratio, which was identical to the standardized uptake value ratio (SUVR).

MRI techniques. Standardized T1, T2, FLAIR, and T2* gradient echo (GRE) MRI was performed for all participants at Samsung Medical Center using the same 3.0T MRI scanner (Philips 3.0T Achieva). T2* GRE-MRI was obtained using the following parameters: axial slice thickness of $5.0 \mathrm{~mm}$, interslice thickness of $2 \mathrm{~mm}$, repetition time of $669 \mathrm{~ms}$, echo time of $16 \mathrm{~ms}$, flip angle of $18^{\circ}$, and matrix size of $560 \times 560$ pixels.

Analysis of CMBs on GRE-MRI. CMBs were defined as lesions $\leq 10 \mathrm{~mm}$ in diameter and were also defined using criteria proposed by Greenberg et al. ${ }^{13}$ Two experienced neurologists who were blinded to patient data such as clinical information, lacunes, WMH, and amyloid burdens reviewed the number and location of CMBs on 20 T2* GRE-MRI axial slices. The 2 neurologists had an agreement kappa value for the presence of CMBs of 0.92 , and a consensus was reached in any initially discrepant cases.

CMBs were counted in 4 lobar regions and also in deep brain regions. Lobar $\mathrm{CMB}$ depicted on the cortical surface model from each group were displayed simultaneously using ParaView software (paraview.org/; Kitware, Clifton Park, NY) as described in appendix e-2.

Lacune and WMH assessment on MRI. A lacune was defined as a lesion $\geq 3 \mathrm{~mm}$ and $\leq 15 \mathrm{~mm}$ in diameter with low signal intensity on $\mathrm{T} 1$ imaging, high signal intensity on T2-weighted imaging, and with a perilesional halo on FLAIR imaging. Two experienced neurologists who were blinded to patient clinical data reviewed the number and location of the lacunes on 80 axial FLAIR slices. The kappa value between the 2 neurologists for the presence of lacunes was 0.78 , and a consensus was reached in any initially discrepant cases.

The extent of WMH on FLAIR was also rated using the semiquantitative scale proposed by Scheltens and colleagues. ${ }^{14}$

Follow-up evaluations with neuropsychological testing, MRI, and PiB-PET. Patients were evaluated annually with a clinical interview, neuropsychological testing, and brain MRI for 3 years. Of the 72 svMCI patients, 65 completed the first year of follow-up, 51 completed the second year, and $52(72.2 \%)$ completed the third year (figure e-1). The dropout rate was $27.8 \%$. Among 72 patients with svMCI, 13 patients developed dementia during the follow-up period. Two patients experienced incident ICH during the 3-year follow-up period.

A total of 50 (69.4\%) patients underwent second PiB-PET at a mean interval of 32.3 months (figure e-1). Those who dropped out were older at baseline than those who completed follow-up $(p=0.024)$ and tended to have higher PiB retention at baseline $(p=0.076)($ table e-1).

Statistical analysis. To evaluate whether the baseline PiB retention ratio and the number of lacunes affect the progression of microbleeds globally or regionally, a generalized estimating equation with a negative binomial distribution was used for analysis after controlling for age, sex, and the baseline $\mathrm{PiB}$ retention ratio or number of lacunes. The interaction between baseline lacune number and $\mathrm{PiB}$ retention ratio on the progression of microbleeds was explored, including with the use of an interaction term ( $\mathrm{PiB}$ retention ratio $X$ number of lacunes) in the above model.

To evaluate whether the $\mathrm{PiB}$ retention ratio or the number of lacunes affects the progression of CMBs, a generalized estimating equation with a negative binomial distribution was used for analysis after controlling for age, sex, and longitudinal measures of the $\mathrm{PiB}$ retention ratio or longitudinal measures of lacunes. To evaluate the effects of WMH severity changes on CMB progression, WMH severity changes were included in the above model instead of longitudinal measures of lacunes. In order to evaluate the interactive effects of longitudinal measures of the $\mathrm{PiB}$ retention ratio and number of lacunes on the progression of microbleeds, an interaction term (longitudinal measures of the $\mathrm{PiB}$ retention ratio $X$ the number of lacunes) was included in the above model.

To evaluate the relationship between $\mathrm{CMB}$ progression and cognitive decline, a generalized estimating equation with a linear model was used with neuropsychological scores as the dependent variables and $\mathrm{CMBs}$ as the independent variables after controlling for age, sex, education, and longitudinal measures of imaging markers (amyloid burden and lacunes). Correction for multiple comparisons was done using the false discovery rate method. SAS software (SAS University Edition; SAS Institute, Cary, $\mathrm{NC)}$ was used for all analyses.

RESULTS Longitudinal measures of lacunes, $\mathrm{PiB}$ retention, and CMBs. In total, 43 of 72 (59.7\%) patients had CMBs at baseline. Over 3 years, 31 of 52 patients $(59.6 \%)$ had incident CMBs, increasing by 68 and 50 in the lobar and deep regions, respectively. Three $(5.8 \%)$ of 52 svMCI patients had CMB counts decreasing by 10 and 9 in the lobar and deep regions, respectively. The number of lacunes increased from $6.8 \pm 7.4$ to $7.9 \pm 8.2$ and PiB SUVR increased from $1.47 \pm 0.38$ to $1.52 \pm 0.45$ in 52 patients monitored over 32.3 months (table 1). Figure 1 illustrates the distribution and number of baseline and incident lobar CMBs in the patient cohort.

Relationship of baseline and longitudinal measures of lacunes and $\mathrm{PiB}$ retention with longitudinal measures of lobar and deep CMBs. Higher lacune number at baseline and longitudinal changes in lacune number were associated with longitudinal increases in lobar $\mathrm{CMB}$ numbers globally and regionally, as well as longitudinal increases in deep CMB numbers (table 2). Higher baseline number or longitudinal increases in $\mathrm{PiB}$ retention were also associated with longitudinal increases in lobar CMB numbers, especially in the parietal, temporal, and occipital regions (table 2). There were interactive effects between baseline and longitudinal lacune number and $\mathrm{PiB}$ retention on longitudinal global, parietal, temporal, and occipital lobar CMB numbers (table 2).

We also analyzed the relationship of CMBs with lacunes or amyloid burden at baseline and followup after controlling for age, sex, and lacune number or $\mathrm{PiB}$ uptake (table e-2). Higher lacune number was associated with presence or higher number of lobar and deep CMBs at both baseline and followup. Neither the presence nor load of lobar/deep $\mathrm{CMBs}$ was associated with baseline or follow-up PiB uptake. 


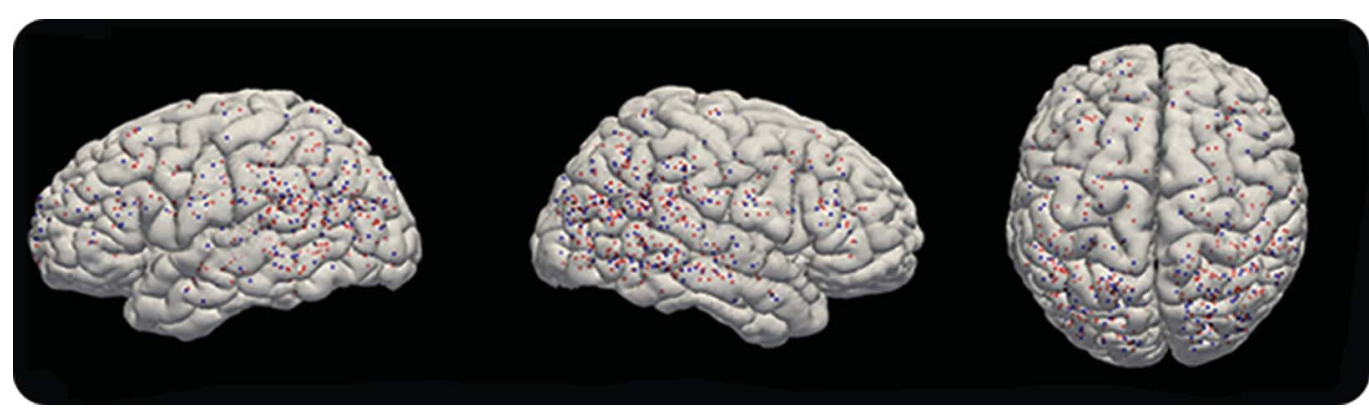

Lobar CMBs from the gradient echo MRI axial scans of all patients were depicted on a single glass brain. Blue dots indicate baseline lobar CMBs and red dots indicate newly developed CMBs.

Increased overall WMH severity tended to be associated with increased deep CMBs $(p=0.059)$ and lobar CMBs in the parietal $(p=0.06)$ and temporal $(p=0.053)$ regions (table 3$)$. Regional WMH severity was associated with regional lobar CMBs in the parietal $(p=0.001)$ and occipital $(p=0.001)$ regions (table 3).

In patients with aMCI without significant $\mathrm{WMH}$, higher lacune number at baseline and longitudinal changes in lacune number were associated with longitudinal increases in lobar CMB numbers (table e-3). Higher lacune number at baseline was also associated with longitudinal increases in deep $\mathrm{CMB}$ numbers (table e-3). PiB retention was not associated with lobar/deep CMBs (table e-3).

Relationship between longitudinal measures of CMBs and neuropsychological results. Increased lobar CMBs were associated with decreased scores on the digit span backward and RCFT copy tests (table 4). There were no significant relationships between longitudinal measures of deep CMBs and decline in neuropsychological scores (table 4).

DISCUSSION Using 3 years of longitudinal amyloid burden and hCSVD data, we investigated the potential pathogenesis of lobar and deep CMB progression. Our major findings are as follows. First, both baseline hCSVD and the progression of hCSVD, as measured by lacune number, contributed to the progression of lobar and deep CMBs. Second, both baseline amyloid deposition and its progression, as measured by $\mathrm{PiB}$ retention, contributed to the progression of lobar, but not deep, CMBs. Third, baseline and progression of hCSVD and amyloid retention synergistically affected the progression of lobar CMBs, especially in the

\begin{tabular}{|c|c|c|c|c|c|c|c|c|c|c|c|c|}
\hline \multirow[t]{4}{*}{ Table 2} & onships bet & ween ba & eline and lon & gitudinal & maging mar & kers and & longitudine & I measur & es of lobar a & and deep & cerebral micr & obleeds \\
\hline & \multicolumn{10}{|c|}{ Longitudinal measures of lobar CMBs } & \multirow{2}{*}{\multicolumn{2}{|c|}{$\begin{array}{l}\text { Longitudinal measures } \\
\text { of deep CMBs }\end{array}$}} \\
\hline & \multicolumn{2}{|c|}{ Lobar, overall } & \multicolumn{2}{|l|}{ Frontal } & \multicolumn{2}{|l|}{ Parietal } & \multicolumn{2}{|l|}{ Temporal } & \multicolumn{2}{|l|}{ Occipital } & & \\
\hline & $\mathrm{B}(\mathrm{SE})^{\mathrm{a}}$ & $p$ & B (SE) & $p$ & B (SE) & $p$ & B (SE) & $p$ & B (SE) & $p$ & B (SE) & $p$ \\
\hline Lacunes & $0.09(0.02)$ & $<0.001$ & $0.11(0.02)$ & $<0.001$ & 0.11 (0.02) & $<0.001$ & $0.08(0.02)$ & 0.001 & $0.07(0.03)$ & 0.008 & $0.11(0.02)$ & $<0.001$ \\
\hline PiB uptake ratio & $1.57(0.58)$ & 0.007 & $-0.12(0.65)$ & 0.850 & $1.97(0.55)$ & $<0.001$ & $1.61(0.66)$ & 0.015 & $2.03(0.63)$ & 0.001 & $-0.52(0.54)$ & 0.333 \\
\hline Interaction $^{b}$ & $0.31(0.06)$ & $<0.001$ & NA & NA & $0.18(0.06)$ & 0.003 & $0.29(0.07)$ & $<0.001$ & $0.43(0.08)$ & $<0.001$ & NA & NA \\
\hline \multicolumn{13}{|l|}{$\begin{array}{l}\text { Longitudinal } \\
\text { measures }\end{array}$} \\
\hline
\end{tabular}

Abbreviations: $\mathrm{NA}=$ not applicable; $\mathrm{PiB}=$ Pittsburgh compound $\mathrm{B}$.

Generalized estimating equation with a negative binomial distribution was conducted using CMBs in each region as the dependent variable and imaging markers (lacunes, PiB retention) as the independent variables after controlling for age and sex.

${ }^{a}$ Values are presented as the regression coefficient (standard error).

${ }^{\mathrm{b}}$ Including an interaction term for lacunes (continuous variable) and PiB retention ratio to covariates of the previous model. 
Table 3 Relationship between longitudinal measures of white matter hyperintensity (WMH) severity and cerebral microbleeds (CMBs)

\begin{tabular}{|c|c|c|c|c|c|c|c|c|c|c|c|c|}
\hline \multirow{3}{*}{$\begin{array}{l}\text { Longitudinal changes } \\
\text { in WMH severity }\end{array}$} & \multicolumn{10}{|c|}{ Longitudinal changes in lobar CMBs } & \multirow{2}{*}{\multicolumn{2}{|c|}{$\begin{array}{l}\text { Longitudinal changes } \\
\text { in deep CMBs }\end{array}$}} \\
\hline & \multicolumn{2}{|c|}{ Lobar, overall } & \multicolumn{2}{|l|}{ Frontal } & \multicolumn{2}{|l|}{ Parietal } & \multicolumn{2}{|l|}{ Temporal } & \multicolumn{2}{|l|}{ Occipital } & & \\
\hline & B (SE) & $p$ & B (SE) & $p$ & B (SE) & $p$ & B (SE) & $p$ & B (SE) & $p$ & B (SE) & $p$ \\
\hline Overall & $0.04(0.03)$ & 0.139 & $0.02(0.03)$ & 0.48 & $0.06(0.03)$ & 0.06 & $0.06(0.03)$ & 0.053 & $0.02(0.03)$ & 0.612 & $0.06(-0.03)$ & 0.059 \\
\hline Frontal & & & $-0.02(0.05)$ & 0.709 & & & & & & & & \\
\hline Occipital & & & & & & & & & $0.35(0.11)$ & 0.001 & & \\
\hline
\end{tabular}

Generalized estimating equation with a negative binomial distribution was used with CMBs as the dependent variable and WMH score as the independent variable after controlling for age, sex, and Pittsburgh compound B uptake ratio. Values are presented as the regression coefficients (standard error).

parietal, temporal, and occipital regions-areas preferentially affected by CAA. Finally, increased lobar CMBs contributed to a decline in attention and visuospatial functions. Therefore, our findings provide insight into the interaction between amyloid burden and hCSVD on the progression of CMBs and cognitive decline.
Our longitudinal study builds upon the results of a prior cross-sectional study, which revealed an interaction between amyloid burden and hCSVD on lobar CMBs. ${ }^{10}$ Specifically, the current longitudinal study showed that baseline amyloid burden and hCSVD predicted the progression of lobar CMBs beyond the degree predicted for either individual marker.

\begin{tabular}{|c|c|c|c|c|c|c|}
\hline \multicolumn{7}{|c|}{ Table $4 \quad$ Relationship between cerebral microbleeds (CMBs) and neuropsychological results } \\
\hline \multirow{2}{*}{$\begin{array}{l}\text { Longitudinal } \\
\text { neuropsychological results }\end{array}$} & \multicolumn{3}{|c|}{ Longitudinal measures of lobar CMBs } & \multicolumn{3}{|c|}{ Longitudinal measures of deep CMBs } \\
\hline & $\mathrm{B}(\mathrm{SE})^{\mathrm{a}}$ & p & Corrected $p$ & $B(S E)^{a}$ & p & Corrected $p$ \\
\hline Digit span forward & $-0.013(0.017)$ & 0.4504 & 0.8366 & $-0.015(0.018)$ & 0.4068 & 0.6509 \\
\hline Digit span backward & $-0.026(0.008)$ & 0.0013 & 0.0104 & $-0.013(0.008)$ & 0.1197 & 0.383 \\
\hline K-BNT & $-0.017(0.146)$ & 0.9080 & 0.978 & $0.042(0.333)$ & 0.8989 & 0.8989 \\
\hline RCFT copy ${ }^{b}$ & $-0.054(0.016)$ & 0.0007 & 0.0104 & $-0.010(0.020)$ & 0.6028 & 0.8037 \\
\hline SVLT immediate recall & $0.003(0.047)$ & 0.9529 & 0.978 & $-0.119(0.053)$ & 0.0235 & 0.188 \\
\hline SVLT delayed recall & $-0.068(0.037)$ & 0.0665 & 0.266 & $-0.069(0.024)$ & 0.0043 & 0.0688 \\
\hline SVLT recognition & $-0.045(0.041)$ & 0.2666 & 0.6094 & $-0.028(0.030)$ & 0.3538 & 0.629 \\
\hline RCFT immediate recall & $-0.036(0.060)$ & 0.5546 & 0.8874 & $-0.024(0.076)$ & 0.7531 & 0.8398 \\
\hline RCFT delayed recall & $-0.098(0.071)$ & 0.1692 & 0.4949 & $-0.081(0.076)$ & 0.2864 & 0.5728 \\
\hline RCFT recognition & $0.006(0.027)$ & 0.8246 & 0.978 & $-0.011(0.024)$ & 0.6604 & 0.8128 \\
\hline COWAT animal & $0.062(0.047)$ & 0.1856 & 0.4949 & $0.026(0.037)$ & 0.4875 & 0.7091 \\
\hline COWAT supermarket & $0.012(0.077)$ & 0.8717 & 0.978 & $-0.066(0.049)$ & 0.1775 & 0.4418 \\
\hline COWAT phonemic & 0.005 (0.173) & 0.9780 & 0.978 & $0.039(0.145)$ & 0.7873 & 0.8398 \\
\hline Stroop color & $-0.183(0.452)$ & 0.6862 & 0.978 & $-0.346(0.266)$ & 0.1933 & 0.4418 \\
\hline K-MMSE ${ }^{\mathrm{b}}$ & $-0.030(0.012)$ & 0.0135 & 0.072 & $-0.025(0.015)$ & 0.0851 & 0.3404 \\
\hline CDR-SOB ${ }^{c}$ & $0.007(0.010)$ & 0.4706 & 0.8366 & $0.015(0.008)$ & 0.0561 & 0.2992 \\
\hline
\end{tabular}

Abbreviations: $\mathrm{CDR}-\mathrm{SOB}=$ Clinical Dementia Rating Sum of Boxes; COWAT $=$ Controlled Oral Word Association Test; K-BNT $=$ Korean version of Boston Naming Test; K-MMSE $=$ Korean version of Mini-Mental State Examination; RCFT = Rey-Osterrieth Complex Figure test; SVLT = Seoul Verbal Learning Test.

Generalized estimating equation with linear modeling was conducted with neuropsychological test scores as the dependent variables and $\mathrm{CMBs}$ as the independent variable after controlling for age, sex, education, and longitudinal measures of imaging markers (Pittsburgh compound B uptake ratio and lacunes). Correction for multiple comparisons was done using the false discovery rate method.

a Values are presented as the regression coefficient (standard error).

${ }^{b}$ Data were transformed using a Box-Cox transformation prior to their inclusion in statistical analyses.

${ }^{\mathrm{c}}$ Data were transformed using a logarithmic transformation prior to their inclusion in statistical analyses. 
Moreover, longitudinal amyloid burden and hCSVD also synergistically affected the progression of lobar CMBs. These findings strongly suggest that lobar CMBs are caused by an arteriopathy related to both hCSVD and cerebral amyloid burden, while the arteriopathy causing deep CMBs is not related to cerebral amyloid burden. The mechanism by which hCSVD and amyloid pathology interact in the development of lobar $\mathrm{CMBs}$ requires further investigation. CAA shows a general predilection for the posterior brain region (similar to the distribution of progressive CMBs in our study), ${ }^{15}$ so it is possible that hCSVD aggravates the deposition of vascular amyloid, or vice versa. One potential pathophysiologic link is the perivascular drainage pathways, an increasingly recognized route for the elimination of interstitial fluid and solutes, including $\beta$-amyloid, from the brain. ${ }^{16}$ Perivascular drainage may be impaired by hCSVD (as indicated by MRI-visible perivascular spaces), leading to impaired amyloid clearance with vascular deposition and more severe CAA with lobar CMB progression. Alternatively, CAA (reflected in increased PiB retention $)^{17}$ is also associated with MRI-visible perivascular spaces in the cerebral hemisphere white matter, ${ }^{18}$ and may lead to the development of white matter small vessel injury, leukoaraiosis, and $\mathrm{CMB}$ progression. ${ }^{17}$ Alternatively, amyloid burden and hCSVD might affect other common pathways, including endothelial dysfunction or inflammation, ${ }^{19}$ which in turn could lead to increased vulnerability to small vessel occlusion or hemorrhage.

One implication of our results is in the selection and risk stratification of patients undergoing antiplatelet or antiamyloid therapy. CMBs are of particular interest because they are an important amyloidrelated imaging abnormality during amyloid immunotherapy in patients with cognitive impairment, ${ }^{20}$ with potential relevance in the prediction of this adverse clinical effect; understanding the mechanisms underlying $\mathrm{CMBs}$ may thus be useful in the design and interpretation of future $\mathrm{AD}$ treatment trials. Whether hCSVD is related to the risk of such complications warrants further study. CMBs have also been implicated as a risk factor for intracerebral hemorrhage following anticoagulant or antiplatelet therapy. ${ }^{21,22}$ Tightly controlling vascular risk factors reduces the progression of hCSVD, which might prevent new-onset $\mathrm{CMBs}$ and the rate of hemorrhagic complications in patients with combined amyloid and hCSVD.

Our findings that both baseline hCSVD and its progression contribute to the progression of lobar and deep CMBs confirm a prior cross-sectional study from our group. ${ }^{10}$ The hypothesis that deep CMBs are associated with hCSVD has also been tested in several longitudinal studies, which consistently showed that a higher baseline burden or progression of hCSVD predicts future deep CMBs. ${ }^{23,24}$ However, previous results regarding incident lobar CMBs were less consistent: 2 longitudinal studies showed that baseline or incident lacunes were associated with incident lobar CMBs, ${ }^{9,25}$ while a previous study did not find a relationship between the progression of hCSVD and incident strictly lobar CMBs. ${ }^{23}$ Our findings support the notion that superficial perforating arteries of pial origin are likely responsible for lobar $\mathrm{CMBs}^{26}$ and may lose their autoregulatory function in the presence of chronic hypertension and hCSVD, ${ }^{27}$ leading to excessive pressure, damage to smooth muscle cells, and microbleeds. ${ }^{26}$ Consistent with 2 other longitudinal studies, ${ }^{9,25}$ our findings imply that lobar CMBs not only reflect CAA.

We found that both baseline amyloid burden and its progression affect the progression of lobar, but not deep, CMBs. Our findings are consistent with a recent CAA study showing that elevated PiB retention at baseline precedes cerebral bleeding, including CMBs. ${ }^{28}$ Another longitudinal study showed that baseline PiB status can be used to predict the incidence of strictly lobar CMBs. ${ }^{25} \mathrm{PiB}$ ligand binds to the fibrillary form of amyloid in both parenchyma and vessel walls. ${ }^{29}$ Increased CAA severity is also reportedly associated with increased neuritic plaques, ${ }^{30}$ although a previous study found that only a quarter of patients with $\mathrm{AD}$ display CAA extensive enough to cause lobar CMBs. ${ }^{31}$ Therefore, increased PiB SUVR might reflect increased CAA, which can lead to the progression of lobar CMBs. It is also reasonable to infer that increased amyloid vasculopathy contributes to the progression of lobar CMBs over time, given that CAA has a predominantly posterior brain distribution. ${ }^{32}$ In fact, several animal studies have provided evidence linking vascular amyloid to disturbances in endothelial-dependent factors, causing disruption of cerebrovascular regulation and extravascular leakage of blood components. ${ }^{33}$

We found that increased lobar CMBs, but not deep CMBs, were associated with decreased scores in attention and visuospatial functions. There has been some controversy regarding the clinical influence of CMBs..$^{24,34,35}$ Some previous studies suggested that CMBs were clinically silent, ${ }^{36}$ while others reported that CMBs were associated with cognitive decline in elderly patients without dementia and ischemic stroke patients. ${ }^{37,38}$ Our longitudinal study demonstrated that longitudinal increases in CMBs affect cognitive decline and evidenced regional specificity in the clinical significance of CMBs. There may be several possible interpretations of our findings regarding the relationship between lobar $\mathrm{CMBs}$ and cognitive impairment in svMCI patients. The relationship between lobar CMBs and cognitive impairment might be explained by CAA, considering that 
CAA is not only a key factor in CMBs, but it has also been associated with cognitive impairment. ${ }^{39}$ Second, CMBs may reflect the effect of co-occurring hCSVD or $\mathrm{AD}$, although the statistical significance remained after controlling for PiB SUVR and lacunes. Finally, the effects of CMBs on cognition may be due to surrounding ischemic changes as well as direct focal damage. However, results from this current study do not fully address these hypotheses. Future studies ought to investigate these complex relationships.

An important strength of this study is its 3-year prospective cohort design using a standardized protocol. Moreover, $70 \%$ of the participants underwent detailed annual neuropsychological testing and structural MRI, with a repeat PiB-PET scan after 3 years. However, there were some limitations. First, although PiB-PET is the most sensitive method available to reliably detect cerebral amyloid in the vessel walls and parenchyma, this modality cannot differentiate these forms of amyloid deposition. Second, we measured longitudinal changes in WMH severity using semiquantitative methods instead of measurements of WMH volume because of technical limitations. However, lacunes are a more specific marker of hCSVD, since WMH may be related to several different pathologic processes. ${ }^{40}$ Third, our svMCI patients were clinically diagnosed via clinical interview, neurologic examination, and neuropsychological testing before they underwent PiB-PET. However, the cognitive symptoms of some svMCI patients with a significant amyloid burden might be driven by $\mathrm{AD}$ pathology. Finally, our study population included a large proportion of patients with significant vascular burden, which may limit the generalizability of our data to other populations.

\section{AUTHOR CONTRIBUTIONS}

Yeo Jin Kim: study design, data analysis, drafting the manuscript. Hee Jin Kim: interpretation of the data, manuscript revision. Jae-Hyun Park: analysis and interpretation of the data. Seonwoo Kim: data analysis. Sook-Young Woo: data analysis. Ki-Chang Kwak: data analysis. Jong Min Lee: data analysis. Na-Yeon Jung: interpretation of the data, manuscript revision. Jae Seung Kim: conceptualization of the study. Yearn Seong Choe: conceptualization of the study. Kyung-Han Lee: conceptualization of the study. Seung Hwan Moon: conceptualization of the study. Jae-Hong Lee: conceptualization of the study. Yun Joong Kim: interpretation of the data. David J. Werring: interpretation of the data, manuscript revision. Duk L. Na: study design, interpretation of the data. Sang Won Seo: study design, interpretation of the data, manuscript revision.

\section{STUDY FUNDING}

This study was supported by the Korean Healthcare Technology R\&D Project (A102065), the KOSEF NRL program grant (MEST; 20110028333), Samsung Medical Center (CRL-108011\&CRS110-14-1), and the Converging Research Center Program through the Ministry of Education, Science, and Technology (2010K001054).

\section{DISCLOSURE}

The authors report no disclosures relevant to the manuscript. Go to Neurology.org for full disclosures.

Received December 3, 2015. Accepted in final form June 28, 2016.

\section{REFERENCES}

1. Alzheimer's Association. 2015 Alzheimer's disease facts and figures. Alzheimers Dementia 2015;11:332-384.

2. Fernando MS, Ince PG; MRC Cognitive Function Ageing Neuropathology Study Group. Vascular pathologies and cognition in a population-based cohort of elderly people. J Neurol Sci 2004;226:13-17.

3. Shah NS, Vidal JS, Masaki K, et al. Midlife blood pressure, plasma beta-amyloid, and the risk for Alzheimer disease: the Honolulu Asia Aging Study. Hypertension 2012; 59:780-786.

4. Lee JH, Kim SH, Kim GH, et al. Identification of pure subcortical vascular dementia using ${ }^{11} \mathrm{C}$-Pittsburgh compound B. Neurology 2011;77:18-25.

5. Lee MJ, Seo SW, Na DL, et al. Synergistic effects of ischemia and beta-amyloid burden on cognitive decline in patients with subcortical vascular mild cognitive impairment. JAMA Psychiatry 2014;71:412-422.

6. Fazekas F, Kleinert R, Roob G, et al. Histopathologic analysis of foci of signal loss on gradient-echo T2*weighted MR images in patients with spontaneous intracerebral hemorrhage: evidence of microangiopathy-related microbleeds. AJNR Am J Neuroradiol 1999;20:637-642.

7. Attems J. Sporadic cerebral amyloid angiopathy: pathology, clinical implications, and possible pathomechanisms. Acta Neuropathol 2005;110:345-359.

8. Vernooij MW, van der Lugt A, Ikram MA, et al. Prevalence and risk factors of cerebral microbleeds: the Rotterdam Scan Study. Neurology 2008;70:1208-1214.

9. Akoudad S, Ikram MA, Koudstaal PJ, et al. Cerebral microbleeds are associated with the progression of ischemic vascular lesions. Cerebrovasc Dis 2014;37:382-388.

10. Park JH, Seo SW, Kim C, et al. Pathogenesis of cerebral microbleeds: in vivo imaging of amyloid and subcortical ischemic small vessel disease in 226 individuals with cognitive impairment. Ann Neurol 2013;73:584-593.

11. Petersen RC, Smith GE, Waring SC, Ivnik RJ, Tangalos EG, Kokmen E. Mild cognitive impairment: clinical characterization and outcome. Arch Neurol 1999;56: 303-308.

12. Ahn HJ, Chin J, Park A, et al. Seoul Neuropsychological Screening Battery-dementia version (SNSB-D): a useful tool for assessing and monitoring cognitive impairments in dementia patients. J Korean Med Sci 2010;25:1071-1076.

13. Greenberg SM, Vernooij MW, Cordonnier C, et al. Cerebral microbleeds: a guide to detection and interpretation. Lancet Neurol 2009;8:165-174.

14. Scheltens P, Erkinjunti T, Leys D, et al. White matter changes on CT and MRI: an overview of visual rating scales: European Task Force on age-related white matter changes. Eur Neurol 1998;39:80-89.

15. Yamada M, Tsukagoshi H, Otomo E, Hayakawa M. Cerebral amyloid angiopathy in the aged. J Neurol 1987;234: 371-376.

16. Carare RO, Bernardes-Silva M, Newman TA, et al. Solutes, but not cells, drain from the brain parenchyma along basement membranes of capillaries and arteries: significance for cerebral amyloid angiopathy and neuroimmunology. Neuropathol Appl Neurobiol 2008;34:131-144.

17. Gurol ME, Viswanathan A, Gidicsin C, et al. Cerebral amyloid angiopathy burden associated with leukoaraiosis: a positron emission tomography/magnetic resonance imaging study. Ann Neurol 2013;73:529-536. 
18. Charidimou A, Jaunmuktane Z, Baron JC, et al. White matter perivascular spaces: an MRI marker in pathologyproven cerebral amyloid angiopathy? Neurology 2014;82: 57-62.

19. Whitehead SN, Cheng G, Hachinski VC, Cechetto DF Progressive increase in infarct size, neuroinflammation, and cognitive deficits in the presence of high levels of amyloid. Stroke 2007;38:3245-3250.

20. Boche D, Zotova E, Weller RO, et al. Consequence of Abeta immunization on the vasculature of human Alzheimer's disease brain. Brain 2008;131:3299-3310.

21. Thoonsen H, Richard E, Bentham P, et al. Aspirin in Alzheimer's disease: increased risk of intracerebral hemorrhage: cause for concern? Stroke 2010;41:2690-2692.

22. Orken DN, Uysal E, Timer E, Kuloglu-Pazarci N, Mumcu S, Forta $\mathrm{H}$. New cerebral microbleeds in ischemic stroke patients on warfarin treatment: two-year follow-up. Clin Neurol Neurosurg 2013;115:1682-1685.

23. Poels MM, Ikram MA, van der Lugt A, et al. Incidence of cerebral microbleeds in the general population: the Rotterdam Scan Study. Stroke 2011;42:656-661.

24. Goos JD, Henneman WJ, Sluimer JD, et al. Incidence of cerebral microbleeds: a longitudinal study in a memory clinic population. Neurology 2010;74:1954-1960.

25. Yates PA, Desmond PM, Phal PM, et al. Incidence of cerebral microbleeds in preclinical Alzheimer disease. Neurology 2014;82:1266-1273.

26. Werring DJ. Cerebral Microbleeds: Pathophysiology to Clinical Practice. Cambridge: Cambridge University Press; 2011.

27. Roman GC, Erkinjuntti T, Wallin A, Pantoni L, Chui HC. Subcortical ischaemic vascular dementia. Lancet Neurol 2002;1:426-436.

28. Yates PA, Sirisriro R, Villemagne VL, et al. Cerebral microhemorrhage and brain beta-amyloid in aging and $\mathrm{Alz}-$ heimer disease. Neurology 2011;77:48-54.

29. Ikonomovic MD, Klunk WE, Abrahamson EE, et al. Postmortem correlates of in vivo PiB-PET amyloid imaging in a typical case of Alzheimer's disease. Brain 2008;131: 1630-1645.
30. Boyle PA, Yu L, Nag S, et al. Cerebral amyloid angiopathy and cognitive outcomes in community-based older persons. Neurology 2015;85:1930-1936.

31. Ellis RJ, Olichney JM, Thal LJ, et al. Cerebral amyloid angiopathy in the brains of patients with Alzheimer's disease: the CERAD experience, part XV. Neurology 1996; 46:1592-1596.

32. Dierksen GA, Skehan ME, Khan MA, et al. Spatial relation between microbleeds and amyloid deposits in amyloid angiopathy. Ann Neurol 2010;68:545-548.

33. Niwa K, Carlson GA, Iadecola C. Exogenous A beta1-40 reproduces cerebrovascular alterations resulting from amyloid precursor protein overexpression in mice. J Cereb Blood Flow Metab 2000;20:1659-1668.

34. Seo SW, Hwa Lee B, Kim EJ, et al. Clinical significance of microbleeds in subcortical vascular dementia. Stroke 2007; 38:1949-1951.

35. Werring DJ, Frazer DW, Coward LJ, et al. Cognitive dysfunction in patients with cerebral microbleeds on T2*-weighted gradient-echo MRI. Brain 2004;127: 2265-2275.

36. Kato $H$, Izumiyama $M$, Izumiyama $K$, Takahashi $A$, Itoyama Y. Silent cerebral microbleeds on T2*-weighted MRI: correlation with stroke subtype, stroke recurrence, and leukoaraiosis. Stroke 2002;33:1536-1540.

37. Yamashiro K, Tanaka R, Okuma Y, et al. Cerebral microbleeds are associated with worse cognitive function in the nondemented elderly with small vessel disease. Cerebrovasc Dis Extra 2014;4:212-220.

38. Gregoire SM, Scheffler G, Jager HR, et al. Strictly lobar microbleeds are associated with executive impairment in patients with ischemic stroke or transient ischemic attack. Stroke 2013;44:1267-1272.

39. Pfeifer LA, White LR, Ross GW, Petrovitch H, Launer LJ. Cerebral amyloid angiopathy and cognitive function: the HAAS autopsy study. Neurology 2002;58:1629-1634.

40. Gouw AA, Seewann A, van der Flier WM, et al. Heterogeneity of small vessel disease: a systematic review of MRI and histopathology correlations. J Neurol Neurosurg Psychiatry 2011;82:126-135.

\section{Share Your Artistic Expressions in Neurology 'Visions'}

AAN members are urged to submit medically or scientifically related artistic images, such as photographs, photomicrographs, and paintings, to the "Visions" section of Neurology ${ }^{\circledR}$. These images are creative in nature, rather than the medically instructive images published in the NeuroImages section. The image or series of up to six images may be black and white or color and must fit into one published journal page. Accompanying description should be 100 words or less; the title should be a maximum of 96 characters including spaces and punctuation.

Learn more at www.aan.com/view/Visions, or upload a Visions submission at submit.neurology.org. 


\section{Neurology}

Synergistic effects of longitudinal amyloid and vascular changes on lobar microbleeds
Yeo Jin Kim, Hee Jin Kim, Jae-Hyun Park, et al.

Neurology 2016;87;1575-1582 Published Online before print September 14, 2016

DOI 10.1212/WNL.0000000000003220

This information is current as of September 14, 2016

\begin{tabular}{|c|c|}
\hline $\begin{array}{l}\text { Updated Information \& } \\
\text { Services }\end{array}$ & $\begin{array}{l}\text { including high resolution figures, can be found at: } \\
\text { http://www.neurology.org/content/87/15/1575.full.html }\end{array}$ \\
\hline Supplementary Material & $\begin{array}{l}\text { Supplementary material can be found at: } \\
\text { http://www.neurology.org/content/suppl/2016/09/14/WNL.0000000000 } \\
\text { 003220.DC1 }\end{array}$ \\
\hline References & $\begin{array}{l}\text { This article cites } 39 \text { articles, } 17 \text { of which you can access for free at: } \\
\text { http://www.neurology.org/content/87/15/1575.full.html\#\#ref-list-1 }\end{array}$ \\
\hline Permissions \& Licensing & $\begin{array}{l}\text { Information about reproducing this article in parts (figures,tables) or in } \\
\text { its entirety can be found online at: } \\
\text { http://www.neurology.org/misc/about.xhtml\#permissions }\end{array}$ \\
\hline Reprints & $\begin{array}{l}\text { Information about ordering reprints can be found online: } \\
\text { http://www.neurology.org/misc/addir.xhtml\#reprintsus }\end{array}$ \\
\hline
\end{tabular}

Neurology ${ }^{\circledR}$ is the official journal of the American Academy of Neurology. Published continuously since 1951, it is now a weekly with 48 issues per year. Copyright () 2016 American Academy of Neurology. All rights reserved. Print ISSN: 0028-3878. Online ISSN: 1526-632X.

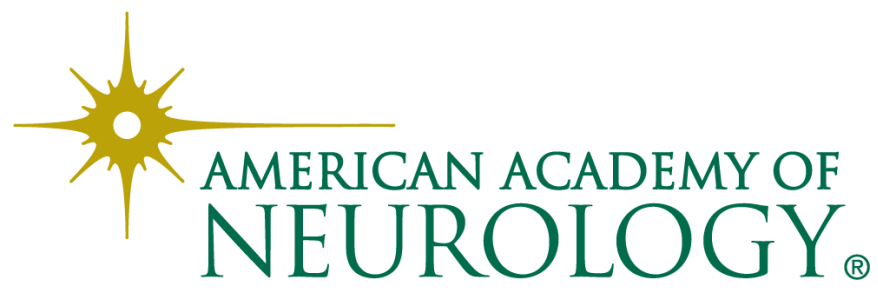

